\title{
Shady: Robust Truss Climbing With Mechanical Compliances
}

\author{
Marsette Vona, Carrick Detweiler, and Daniela Rus \\ Massachusetts Institute of Technology, Cambridge, Massachusetts, USA. \\ \{vona, carrick, rus\}@csail.mit.edu
}

\section{Motivation}

Many large terrestrial structures - towers, bridges, construction scaffolds - are sparse assemblies of rigid bars connected together at structural nodes. This is also true of many in-space structures such as antennae, solar panel supports, and space-station members. A long-term application of truss climbing robots is automated assembly, repair, and inspection of such truss-like structures: one or more climbing robots could grip the bars and locomote about the truss, conveying sensors, tools, or construction materials. The robot could then either carry out the desired task on its own or cooperate with a human $[1,7]$.

Truss climbing is a special case of structure climbing, with some particular challenges. Many previous structure climbing robots, e.g. as in Pack et al [9], and others referenced therein, are intended to climb on assemblages of $2 \mathrm{D}$ planar surfaces. Only a few structure climbing robots, such as the mechanism described by Nechba et al in [7], are also designed for climbing on truss-like structures where the members are more nearly $1 \mathrm{D}$ links.

The penalties for uncertainty are potentially higher for truss climbing than for climbing on planar surfaces. Consider foot placement. On a large 2D surface, foot placement can be resilient to significant parallel-plane misalignment, usually does not require strong certainty of the perpendicular distance to the surface (as the foot can often be extended until it hits the surface), and is similarly tolerant of orientation uncertainties. However, the comparable task in truss climbing - gripping a thin structural member starting from a nearby but uncertain spatial pose - can be much more sensitive: even small translation and orientation misalignments can result in a weak or missed grip.

We propose a minimalist mechanical structure with several specific and intentional mechanical compliances (springs), detailed below, to address these challenges. Combined with wide-opening grippers and proprioceptive control algorithms, also detailed below, we hypothesized that these compliances could allow significant translation and orientation misalignment and still succeed in a firm and certain grip. 
We constructed a robot called Shady to test this theory, and also to serve a very particular purpose, motivated by need: window shading. Our group works in a lab with a large wall-window, about $4 \mathrm{~m}$ tall and $8 \mathrm{~m}$ wide (figures 1 and 2), which has no shades to block sunlight. Instead of traditional shades which would block the whole window, Shady locomotes on the truss-like aluminum window frame and deploys a $0.6 \mathrm{~m}$ diameter mylar sun-shade, thus providing active personal shading without significantly decreasing ambient light.

We have performed experiments indicating that the current version of the Shady hardware, which implements our proposed mechanical compliances and control algorithms, is over $99.8 \%$ successful at completing locomotion primitives (described below) on our lab's unmodified window frame. Only two non-dangerous faults occurred out of over 1296 movements comprising several long climbing sequences, exercising all types of motion for the robot. A prior version of the hardware, which employed algorithms based on data from contact and proximity sensors in lieu of specific compliances and proprioception, was only about $80 \%$ successful at a corresponding set of primitives and required a modified window frame [2].

Several truss climbing robots have been explored by other groups, e.g. Staritz et al's "Skyworker" [8], Amano et al's handrail-gripping robot for firefighting [4], Ripin et al's pole climbing robot [11], Nechba, Xu, Brown et al's "mobile space manipulator SM2" [7], Kotay and Rus' "Inchworm" [5], and Almonacid et al's parallel mechanism for climbing on pipe-like structures [6]. This paper presents a new mechanical design and novel control using intentional mechanical compliances and proprioception, with experimentally confirmed robustness.

\section{Technical Approach}

Shady is a bilaterally-symmetric mechanism with two rotating grippers and a central circular deployable sun-shade (figures 1 and 2). Truss locomotion proceeds as a series of grip-rotate-grip steps (figure 1, left). At present, Shady is only capable of locomoting on planar trusses like our lab's window frame; however our group is also currently developing a similar robot with an additional central twist DOF for climbing full 3D truss structures [10].

Shady's grippers (figure 3) are symmetric 6-bar linkage mechanisms situated in rotating "barrels". Actuated through central 50:1 worm gears, the grippers open to over $7 \mathrm{~cm}$ in about $5 \mathrm{~s}$ and close on the $2.5 \mathrm{~cm}$ window bar in about $15 \mathrm{~s}^{1}$. Each 6-bar is actually two coupled 4-bars: 0-1-2-5 and 2-3-4-5 (link 5 is the barrel), and these are both in singularity when the gripper is closed on a window bar, resulting in very large mechanical advantage and effectively zero backdriveablity at closure. Silicone rubber grip pads develop over $46 \mathrm{~N}$ measured compression force against the window frame with very high stiction

\footnotetext{
${ }^{1}$ Closing takes longer due to the grip refinement algorithm, described later.
} 


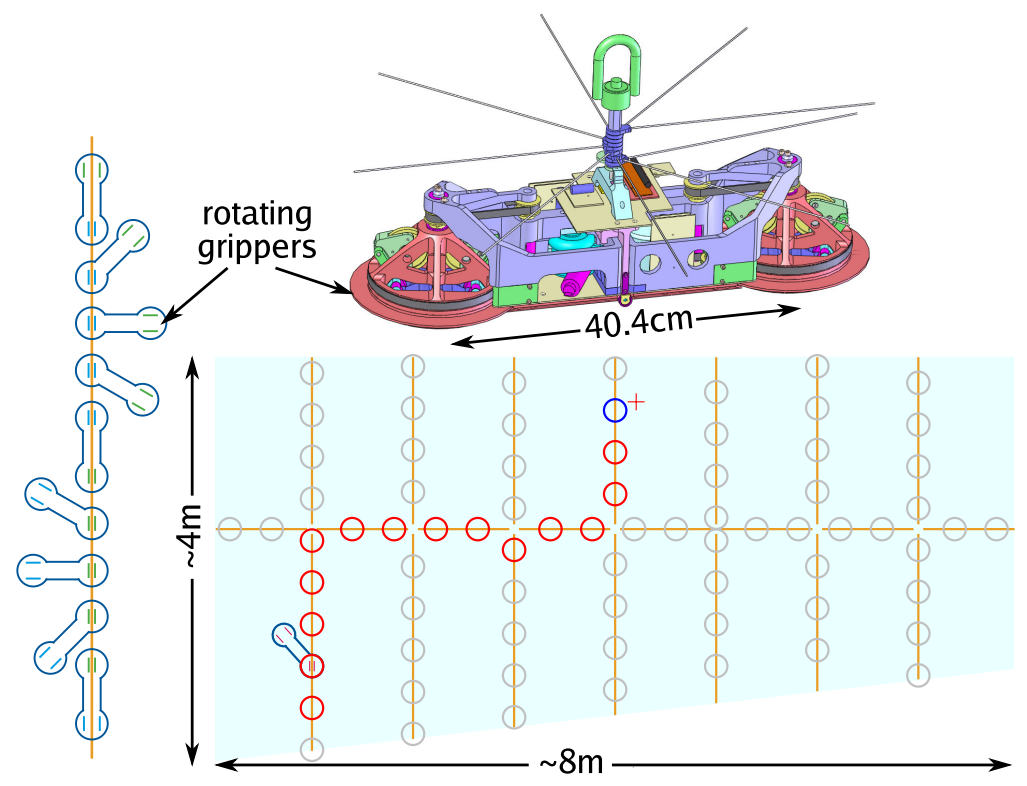

Fig. 1. CAD representation of Shady (mylar shade not shown), locomotion scheme on a straight truss member, and our lab's trapezoidal wall-window with a set of grip points reachable from a starting pose (light circles) and a locomotion path (dark circles) to a commanded target location.

and no measurable slip when closed. When fully opened, the gripper pads retract behind the bounding plane of the barrel, allowing the barrel to brush past the window frame without collision.

Shady measures $40.4 \mathrm{~cm}$ between barrel centers $(59.4 \mathrm{~cm}$ end-to-end), a scale selected to match the pitch of the window-frame truss. Barrel rotations are effected at about $10^{\circ} / \mathrm{s}$ by a series-elastic belt drive actuator incorporating a non-backdriveable worm gear (figure 5), leading to a maximum lineal locomotion speed of

$$
\frac{\text { one center-to-center body length }}{\text { ungrip time }+180^{\circ} \cdot 1 \mathrm{~s} / 10^{\circ}+\text { grip time }}=\frac{40.4 \mathrm{~cm}}{5 \mathrm{~s}+18 \mathrm{~s}+15 \mathrm{~s}} \approx 1 \frac{\mathrm{cm}}{\mathrm{s}},
$$

which is acceptable in practice for this application - Shady can climb from the bottom to the top of the window in under seven minutes, and the apparent position of the sun moves much more slowly ${ }^{2}$. Shady extends about $15 \mathrm{~cm}$ (24cm including shade mechanism and belay hook) outwards from the window frame, and weighs $3.50 \mathrm{~kg}$. Most of the mechanical components are made of ABS plastic formed on a rapid-prototype machine, and the largest of these are hollow to reduce mass. Shady incorporates four $3.7 \mathrm{~A}-\mathrm{H} \mathrm{Li}-\mathrm{Po}$

\footnotetext{
${ }^{2}$ Also, the primary design goal for Shady is robustness, not speed.
} 


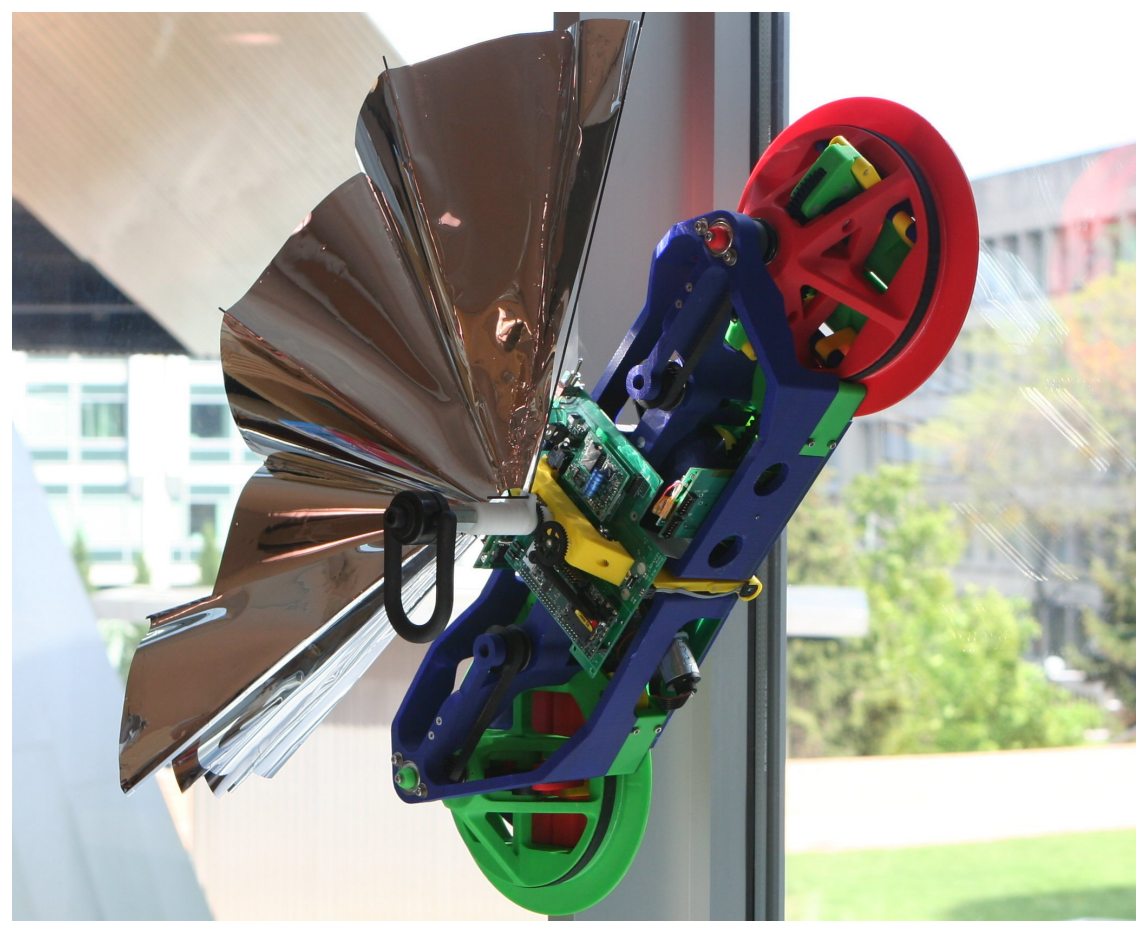

Fig. 2. Shady with circular mylar shade partially deployed.

batteries sufficient for over 6 hours of continuous un-tethered climbing on a single charge, five in-house motor control boards which run low-level feedback

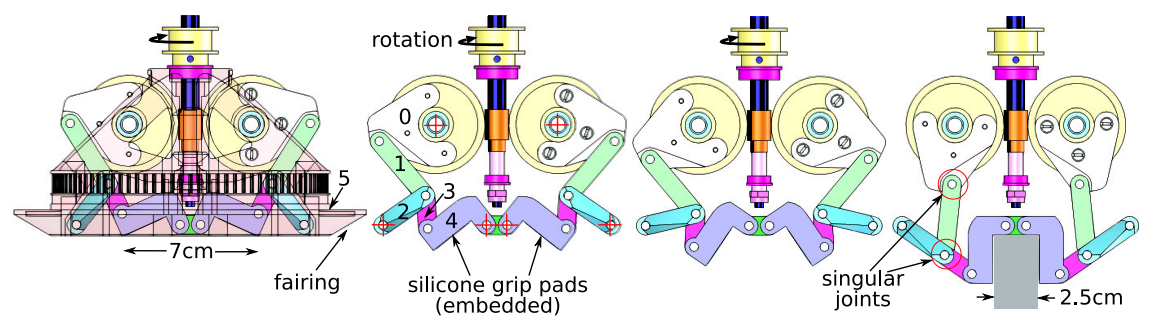

Fig. 3. Motion sequence showing the symmetric 6-bar gripper linkages (actually coupled 4-bar pairs) closing on a window frame member, actuated by rotation of a central worm gear. Link 5, the barrel, is shown only in the initial step; red crosses in the second picture indicate the locations of pins fixing links 0,2 , and 4 to the barrel. When closed, the two four bar linkages which make up the 6 bar are both in singularity (circled joints in-line). 
control loops, a top-level real-time processor, and a miniature Bluetooth ${ }^{\circledR}$ wireless modem for communication with a command and control workstation. The barrel rotation and gripper actuators are based on 6V Maxon A-Max 22 brushed DC motors with integral 19:1 planetary gearboxes, and the fan is actuated by a small Sanyo 12GN-NA4S DC gearmotor.

\subsection{Mechanical Compliances}

Shady contains three intentional mechanical compliances (springs): a central hinge with about $+6^{\circ},-1^{\circ}$ maximum travel (figure 4) that biases the grippers towards the window frame, and two actuator torsion mounts composed of antagonistic pairs of compression springs which enable about $\pm 3^{\circ}$ deflection on each barrel rotation (figure 5). Potentiometers measure the motion at

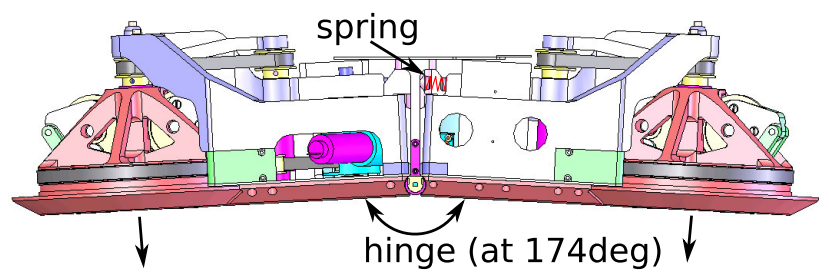

Fig. 4. Central sprung hinge, shown extended $3^{\circ}$ downwards on each side (the maximum travel), biases the grippers towards the window frame (shade mechanism not shown).

each compliance, and this proprioceptive knowledge is incorporated into the control algorithms as described below.

The antagonistic pair of springs on the barrel rotation motor, which is otherwise free to rotate about the same axis as the timing pinion that it drives through a worm gear, forms a series-elastic actuator ([3]): the compression of the springs as measured by the potentiometer is directly proportional to the actuator's applied torque, and can be used in a feedback loop to control that torque. Commanding zero torque enables us to selectively turn the normally non-backdriveable barrel rotation actuator into a freely backdriveable mechanism (up to the saturation limits of the motor), useful in handling/configuring the robot, and a critical component of the grip refinement algorithm. We also utilize this torque control when preloading the mechanism to avoid sag due to gravity, as described below. 

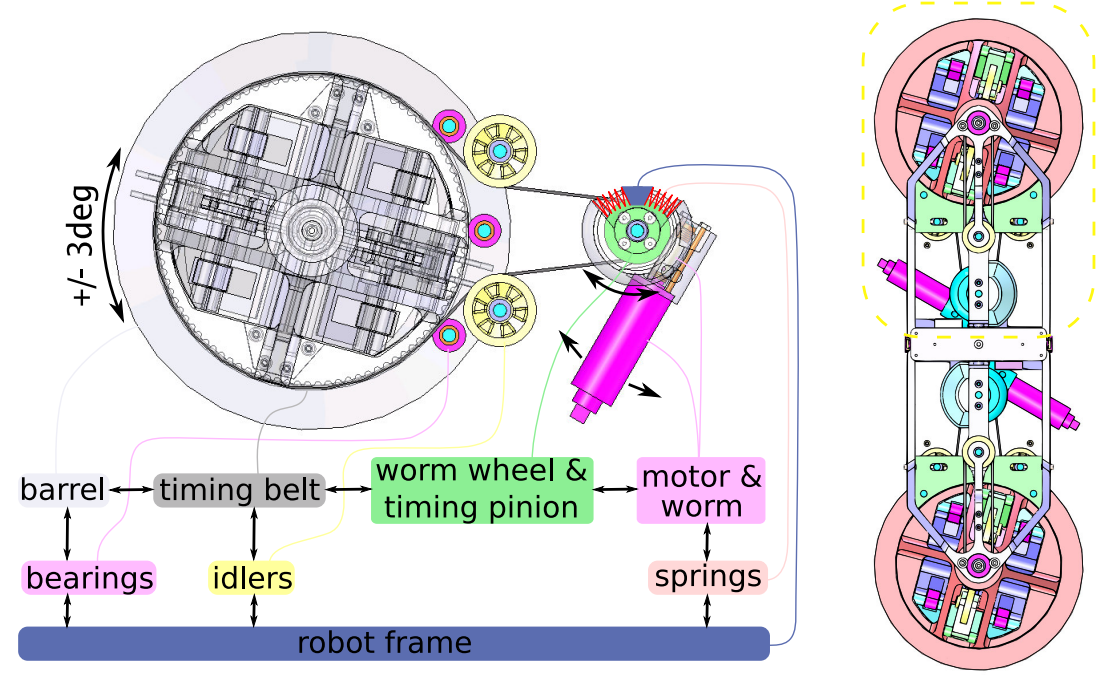

Fig. 5. Series-elastic barrel rotation actuator with torsion-mounted motor. Block diagram indicates force/torque transmission among the various elements. Maximum deflection is about $\pm 3^{\circ}$ of barrel rotation.

\subsection{Grip-Refinement Algorithm}

A major concern in truss-climbing is grip failure due to uncertainty, i.e., falling off the truss. For a planar truss climber like Shady ${ }^{3}$, this can occur when in-plane pose uncertainty of the connecting gripper is beyond the in-plane misalignment (figure 6, left) tolerance of the grip mechanism, or alternately it could be due to a cumulative process where successive grips "walk" the robot normal to the plane of the truss. This walk-off effect may in some cases be mechanically avoidable by designing the gripper to partially or fully envelop the cross-section of a truss member. In practice, however, some trusslike structures effectively present a fully convex cross-section which prevents an enveloping grasp (figure 6), and our laboratory window is such a case. We have also noted that there can be some "cross-talk" between these two mechanisms: it appears that when the gripper is not well-centered to start, the (intentionally) high stiction of the silicone rubber grip pads can cause them to "catch" on the vertex of the window bar which is encountered first, preventing the gripper from fully sliding down onto the bar (figure 6, right).

Algorithm 1, GRIP-REFINEMENT, leverages the central hinge and barrel rotation compliances to minimize these effects. GRIP-REFINEMENT is executed each time a gripper is closed. This algorithm can be considered proprioceptive, as it uses the barrel rotation springs not only to permit deflection but also to measure that deflection and the torque causing it. The third compliance in the

\footnotetext{
${ }^{3}$ We hypothesize that our analysis has a simple extension to full $3 \mathrm{D}$ truss climbing.
} 


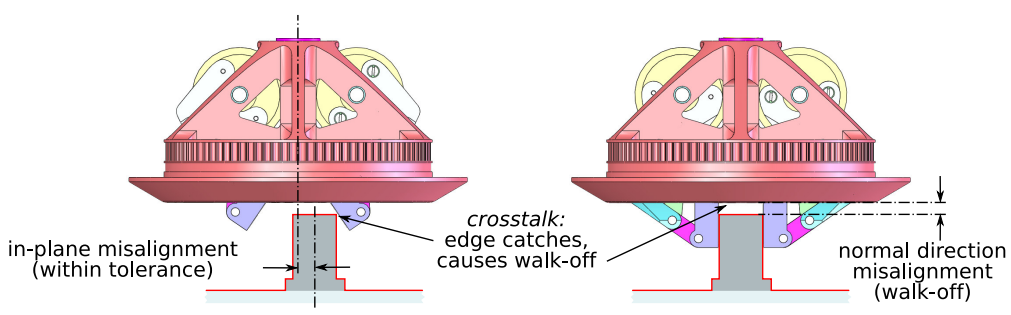

Fig. 6. In-plane gripper misalignment, normal direction misalignment (walk-off), and the crosstalk effect. Convex cross-section formed by truss and window glass, highlighted, prevents a mechanically enveloping grip.

GRIP-REFINEMENT $(g)$

1 Let $o$ be the other gripper, and measure its barrel's initial torque $T_{o}$.

2 Close $g$ completely.

3 Command $o$ 's barrel to torque $T_{o}$ and $g$ 's barrel to zero torque.

4 Measure the actual angular positions of both barrels $P_{g}$ and $P_{o}$.

5 Command barrel rotation actuators to hold positions $P_{g}$ and $P_{o}$.

6 Re-open $g$ to $50 \%$ of the fully open state.

7 Re-close $g$ at $20 \%$ speed.

8 Command both barrels to zero torque.

Algorithm 1: Grip refinement using mechanical compliances and proprioception. Gripper $g$ is currently open and has been commanded to close. The other gripper is implicitly already closed, and is initially supporting the robot. The controllers seek to maintain either torque or position, whichever was most recently commanded.

robot - the sprung central hinge - also comes into play, as it provides a bias force to push the closing gripper towards the window once it is aligned. While we have proprioceptive sensors on the hinge, our algorithms do not yet use them. In the future we may incorporate this information for checking whether the gripper is actually in contact with the window frame. To that end, we are also considering adding a validation step at the end of GRIP-REFINEMENT, where we will command a small torque on the barrel of the just-attached gripper - if it were to actually rotate, it must have closed on thin air.

GRIP-REFINEMENT appears crucial to robust operation of the robot-a large proportion of the over 320 grips we have observed were initially offset in the normal direction (i.e. walked-off) by up to about $8 \mathrm{~mm}$ due to the abovementioned cross-talk effect. In virtually all cases, GRIP-REFINEMENT reduces this walk-off to less than about $2 \mathrm{~mm}$, and usually to $0 \mathrm{~mm}$. 


\subsection{Avoiding Sag Under Gravity}

The plane of our window frame truss is vertical (as with most windows), so gravity is an important design consideration. The effect is minimal when in double support (both grippers attached) but requires special consideration in single support - unless the robot body is pointing either straight up or straight down, gravity will induce a static torque on the connected barrel rotation with a magnitude dependent on the angle of the body. If unaccounted, this torque would cause Shady to sag due to deflection in the rotation actuator springs. We use the following procedure to preload the springs and avoid this sag:

- Shady is always "launched" (i.e. initialized) in double support on a vertical bar, and the most-recent single support torque $T_{s}$ is initialized to 0 .

- Whenever the robot leaves double support (opens a gripper), the barrel $b$ of the remaining connected gripper is commanded to a preload torque $T_{p}$ where $T_{p}=T_{s}$ if $b$ was the prior single-support barrel, and $T_{p}=-T_{s}$ otherwise. Once the gripper is opened, $b$ is commanded to hold position rather than torque, as torque will vary if $b$ is commanded to rotate.

- Whenever the robot enters double support (closes a gripper), the actual torque on the barrel containing the already-closed gripper (i.e. the current single-support barrel) is measured and saved in $T_{s}$.

This particular method of accounting $T_{s}$ does not require explicit knowledge of any absolute orientation relative to the gravity vector, and will thus be immune to uncertainty in measurement or estimation thereof. Under this preload method, virtually all the movements we have observed show no perceptible sag upon gripper opening, independent of orientation.

\section{Experiments and Results}

We have performed three types of experiments on the Shady hardware. First, we tested GRIP-REFINEMENT with successively greater in-plane offsets to determine the maximum tolerable misalignment (section 3.1). Next, we placed Shady on the window frame and commanded a cyclic climbing trajectory which exercised the grip, ungrip, and rotate motion primitives in all possible orientations (section 3.2). Finally we commanded locomotion sequences out-and-back across the window, similar to that depicted in the lower right of figure 1 (section 3.3). In total, over 1296 individual grip, ungrip, and rotate motions were executed, with only two failures (other than the grips which intentionally failed in the first test set), a reliability rate of over $99.8 \%$. The two faults which did occur were not dangerous: the robot simply stopped and informed the operator that an unexpected state was encountered. We believe that in both cases the most likely cause was an intermittent fault in one encoder interface circuit. The command/control software permitted us to investigate this problem over the RF link to the robot, to remotely re-initialize that 
encoder circuit, and then to resume normal operations, all without requiring physical access to the robot.

\subsection{Gripper Misalignment Tests}

Using the GRIP-REFINEMENT algorithm, we conducted an experiment to determine the maximum misalignments that can be tolerated before a grip fails. In this context, the connected barrel is gripped on the window frame, and the distal barrel is initially ungripped. Two tests were performed. In the first, we kept the distal gripper aligned to the robot body and we rotated the connected barrel in $1^{\circ}$ increments, commanding the distal barrel to grip at each step. The maximum tolerated misalignment in this situation is about $3^{\circ}$, which corresponds to about $2.1 \mathrm{~cm}$ horizontal displacement at the distal barrel: $(40.4 \mathrm{~cm})\left(\sin 3^{\circ}\right) \approx 2.1 \mathrm{~cm}$, giving a healthy $4.2 \mathrm{~cm}$ lateral uncertainty tolerance band for gripping. The range of compliance is related to the maximum opening width of the gripper, about $7 \mathrm{~cm}$, as compared with the $2.5 \mathrm{~cm}$ window bar: $(7 \mathrm{~cm}-2.5 \mathrm{~cm}) / 2=2.25 \mathrm{~cm}$. In the second test, we held the connected barrel fixed and incrementally rotated the distal barrel. Up to $13^{\circ}$ misalignment is tolerated here, giving a significant $26^{\circ}$ tolerance band. Though these grip tests were performed with the robot in a vertical configuration, we expect the performance to be similar for other orientations.

\subsection{Grip and Rotate Primitive Tests (Cyclic Climbing)}

We designed a simple cyclic locomotion sequence which takes the robot one full cycle around a structural node of the window frame in 48 grip/ungrip/rotate motions, leaving it where it started. This sequence is designed to test each motion in all of the orientations that would be encountered on our window frame. We ran it for almost 5 full loops around the node, a total of 984 primitive motions over nearly 5 hours. The experiment was interrupted by human intervention only twice due to the two faults described above. No significant normal-direction misalignment (walk-off) was observed at the end of the run, nor was any significant in-plane (i.e. odometric) shift apparent.

\subsection{Out-and-Back Locomotion Sequences}

As a final test, we commanded the robot to repeatedly climb the window from a starting position near the bottom to a point near the top, deploy the fan, retract it, and then to return to the starting position. The overall sequence was similar to that depicted at the lower right in figure 1 . We ran this experiment over four full cycles, a total of 312 grip/ungrip/rotate primitive motions, with no locomotion faults and no human intervention. Again, there was no significant walk-off after the experiment, however the robot appeared to have shifted about $3-5 \mathrm{~mm}$ in-plane along the truss due to odometry error. 


\section{Summary and Conclusions}

We have demonstrated $99.8 \%$ robust un-tethered climbing, including several long uninterrupted autonomous climbs, on an unmodified truss-like structure that is part of our lab's building. While many other structure-climbing robots have been reported, most attach to broad flat walls; the penalties for uncertainty can be higher in a truss, as the nearly 1-dimensional structural elements can be difficult to grasp reliably. We hypothesized that a particular set of intentional mechanical compliances would allow us to achieve robust truss climbing, a theory which has been supported by our experiments. A previous version of our robot which did not incorporate these compliances, instead using sevaral types of sensors, was only about $80 \%$ robust, primarily due to unhandled sensor noise, excessive pose uncertainty, and related problems.

\section{References}

1. Ben Iannotta. Creating robots for space repairs. Aerospace America, pages 36-40, May 2005.

2. Carrick Detweiler, Marsette Vona, and Daniela Rus. Hierarchical control for self-assembling mobile trusses with passive and active links. In IEEE ICRA, 2006.

3. G. Pratt and M. Williamson. Series elastic actuators. In Proceedings of IEEE International Conference on Intelligent Robots and Systems, pages 399-406, 1995.

4. Hisanori Amano, Koichi Osuka, and Tzyh-Jong Tarn. Development of vertically moving robot with gripping handrails for fire fighting. In IEEE/RSJ International Conference on Intelligent Robots and Systems, pages 661-667, Maui, HI, 2001.

5. Keith D. Kotay and Daniela L. Rus. Navigating 3d steel web structures with an inchworm robot. In IEEE International Conference on Intelligent Robots and Systems, 1996.

6. M. Almonacid, R. J. Saltarén, R. Aracil, and O. Reinoso. Motion planning of a climbing parallel robot. IEEE Transactions on Robotics and Automation, 19(3):485-489, 2003.

7. Michael Nechyba and Yangsheng Xu. Human-robot cooperation in space: SM2 for new space station structure. IEEE Robotics and Automation Magazine, 2(4):4-11, Dec. 1995.

8. Peter J. Staritz, Sarjoun Skaff, Chris Urmson, and William Whittaker. Skyworker: A robot for assembly, inspection and maintenance of large scale orbital facilities. In IEEE ICRA, pages 4180-4185, Seoul, Korea, 2001.

9. Robert T. Pack, Joe L. Christopher, and Kazuhiko Kawamura. A rubbertuatorbased structure-climbing inspection robot. In IEEE ICRA, Albuqurque, New Mexico, Apr. 1997.

10. Yeoreum Yoon. Modular robots for making and climbing 3-d trusses. Master's thesis, Massachusetts Institute of Technology, June 2006.

11. Zaidi Mohd Ripin, Tan Beng Soon, A.B. Abdullah, and Zahurin Samad. Development of a low-cost modular pole climbing robot. In TENCON, volume I, pages 196-200, Kula Lumpur, Malaysia, 2000. 